Pacific Journal of Mathematics

HOMOMORPHISMS OF MINIMAL FLOWS AND Da 


\title{
HOMOMORPHISMS OF MINIMAL FLOWS AND GENERALIZATIONS OF WEAK MIXING
}

\author{
Douglas McMahon AND TA-Sun Wu
}

In this paper we are concerned with generalizations of weakly mixing. Let $\phi:(X, T) \rightarrow(Y, T)$ be a homomorphism of metric minimal flows and let $S(\phi)$ denote the relativized equicontinuous structure relation. The main result is that if $\phi$ has a $\operatorname{RIM}, \lambda$, and $z \in Z$ such that the support of $\lambda_{z}$ equals the fiber $X_{0}=\phi^{-1}(z)$, then:

$$
o c\left(V_{1} \times \cdots V_{n}\right) \supseteq S(\phi)\left(V_{1}\right) \times \cdots \times S(\phi)\left(V_{n}\right),
$$

and also there exists a dense set of points $x_{1}, x_{2}, x_{3}, \ldots$ in $X_{0}$, such that $o c\left(x_{1}, x_{2}, x_{3} \ldots\right) \supseteq S(\phi)\left(x_{1}\right) \times S(\phi)\left(x_{2}\right) x \ldots$

0. Introduction. This paper is chiefly concerned with homomorphisms of minimal flows (on compact Hausdorff spaces by a discrete phase group) having relative invariant measures (RIM's). If $\phi: X \rightarrow Z$ has a RIM, $\lambda$, we will frequently restrict our attention to points $z$ in $Z$ with the support of $\lambda_{z}$ equal to $\phi^{-1}(z)$ since otherwise the results would be substantially more difficult to state (and prove).

The major motivation for this paper is a generalization of weakly mixing - if $(X, T)$ is a metric minimal flow having an invariant measure, then it is well known that $Q=X \times X$ implies $\operatorname{cls}\left(x, x^{\prime}\right) T=X \times X$ for some $x, x^{\prime}$ in $X$; we show that even when $Q \neq X \times X$ a similar statement holds, that $\operatorname{cls}\left(x, x^{\prime}\right) T \supseteq Q(x) \times Q\left(x^{\prime}\right)$ for some $x, x^{\prime}$ in $X$. The main results of this paper are generalizations of this idea. Some may also be viewed as a study of the recurrence properties of various subsets of $X$. We will now mention some special cases of the main results.

Suppose $\phi: X \rightarrow Z$ has a RIM, $\lambda$, and $X, Z$ are minimal and metric. Then $S(\phi)=\left\{\left(x, x^{\prime}\right):\left(x u, x^{\prime} u\right) \in Q(\phi)\right.$ for some $\left.u \in J\right\}$ (see 2.1). Suppose $z \in Z$ such that the support of $\lambda_{z}$ equals the fiber $X_{0}=\phi^{-1}(z)$. If $N=o c\left(V_{1} \times \cdots V_{n}\right)$ where $V_{i}$ is an open set in $X_{0}$, then $N \supseteq S(\phi)\left(V_{1}\right) \times$ $\cdots \times S(\phi)\left(V_{n}\right)$ (see 1.1). Also there exists a dense set of points $x_{1}, x_{2}$, $x_{3} \cdots$ in $X_{0}$ such that $o c\left(x_{1}, x_{2}, x_{3}, \ldots\right) \supseteq S(\phi)\left(x_{1}\right) \times S(\phi)\left(x_{2}\right) \times \ldots$, (see 1.5). If $R$ is the smallest closed invariant equivalence relation containing $\left(x_{1}, x_{2}\right), x_{1}, x_{2}$ as above, then $\phi^{\prime}: X / R \rightarrow Z$ is almost automorphic, that is, $Q\left(\phi^{\prime}\right)(y)=\{y\}$ for some $y$ in $X / R$ (see 1.4). If $N=$ $o c\left(\{x\} \times V_{1} \times \cdots \times V_{n}\right)$ where $V_{1}, \ldots, V_{n}$ are open sets in $X_{0}$, then $N \supseteq$ $S(\phi)(x) \times V_{1} \times \cdots \times V_{n}$ (see 2.9) and $N \supseteq S(\phi)(x) V \times S(\phi)\left(V_{1}\right) v \times$ $\cdots \times S(\phi)\left(V_{n}\right) v$ for every $v$ in $J$ (see 2.11). In part we showed the last statement as a possible start in determining whether or not for each $x$ in 
$X_{0}$ there exists $x^{\prime}$ in $X_{0}$ with $\operatorname{cls}\left(x, x^{\prime}\right) T \supseteq S(\phi)(x) \times S(\phi)\left(x^{\prime}\right)$. If $x_{l}, y_{l} \in$ $X_{0}$ and $x=\left(x_{l}\right) \in \Pi X, y=\left(y_{l}\right) \in \Pi X$, then $(x, y) \in Q(\Pi \phi)$ iff $\left(x_{l}, y_{l}\right)$ $\in Q(\phi)$ for every $i$, (see 2.13$)$.

Definitions and Notation. Let $(X, T)$ be a flow with compact Hausdorff phase space $X$ and discrete phase group $T$. We will write $X$ for both the flow and the phase space. Suppose $\Phi: X \rightarrow Z$ is a homomorphism of flows. We will assume $\phi$ is onto. We denote the orbit closure of $x$ by $o c(x)(=\operatorname{cls}(x T))$. We let $X_{m}$ denote the set of transitive points (points with dense orbit), $R_{m}(\phi)=\left\{\left(x, x^{\prime}\right) \in X_{m} \times X_{m}: \phi(x)=\phi\left(x^{\prime}\right)\right\}$, $Q_{m}(\phi)=\left\{\left(x, x^{\prime}\right)\right.$ : there exist nets $t_{n}$ in $T$ and $\left(x_{n}, x_{n}^{\prime}\right) \in R_{m}(\phi)$ such that $\left(x_{n}, x_{n}^{\prime}\right) \rightarrow\left(x, x^{\prime}\right)$ and $\left.\left(x_{n}, x_{n}^{\prime}\right) t_{n} \rightarrow\left(x_{0}, x_{0}\right)\right\}$ for any $x_{0}$ in $X_{m}, S_{m}(\phi)$ is the smallest closed (in $R_{m}(\phi)$ ) invariant equivalence relation containing $Q_{m}(\phi)$.

When $X$ is minimal $X_{m}=X, R_{m}(\phi)=R(\phi), Q_{m}(\phi)=Q(\phi)$ is the relativized regionally proximal relation $S_{m}(\phi)=S(\phi)$ is the relativized equicontinuous structure relation. If $X$ is minimal and $Z$ is the singleton flow, we denote $Q(\phi)$ by $Q$ and $S(\phi)$ by $S$. Let $P$ denote the proximal relation on any minimal flow.

Neighborhoods are assumed to be open, we denote the set of neighborhoods of $x$ by $\Re_{x}$. The Stone-Čech compactification of $T$ is denoted by $\beta T, M \subseteq \beta T$ denotes the universal minimal set (a minimal right ideal in $\beta T), J \subseteq M$ denotes the set of idempotents in $M$.

The set of closed subsets of $X$ is denoted by $2^{X}$ and is given the usual Hausdorff topology. For $A \in 2^{X}, p \in \beta T$, we denote the limit in $2^{X}$ of $A t_{n}$ by $A \circ p$, where $t_{n} \rightarrow p$ in $\beta T ; A p=\{a p: a \in A\}$. A homomorphism of minimal flows, $\phi: X \rightarrow Y$, is relatively incontractible (RIC) iff for every $p \in M, \Phi^{-1}(y p)=\left(\phi^{-1}(y) u\right) \circ p$ where $y \in Y, u \in J$ with $y u=y$ (see $\left[\mathbf{5}_{\mathbf{b}}\right]$ for details).

Let $\mathscr{N}(X)$ be the set of Borel probability measures on $X$. For $\mu$ in N( $(X)$ define $\mu t$ by $\mu\left(A t^{-1}\right)$ for every measurable set $A$. A RIM (relative invariant measure - also called a section) $\lambda$ for $\phi: X \rightarrow Z$ is a homomorphism $\lambda: Y \rightarrow \mathscr{N}(X)$ such that the support of $\lambda$ is contained in the fiber $\phi^{-1}(z)$. If $z$ is fixed, then for any RIM, $\lambda, S_{\lambda}$ denotes the support of $\lambda_{z}$. Also we define $\hat{\varphi}: ~ \Re(X) \rightarrow \Re(Z)$ by $\hat{\alpha}(\mu)(A)=\mu\left(\phi^{-1}(A)\right), A$ a measurable subset of $Z$. For $B \subseteq \Re(X)$ we denote the closed convex hull of $B$ by $\overline{\operatorname{co}}(B)$.

Given $\varphi: X \rightarrow Z, \theta: Y \rightarrow Z, X \circ^{Z} Y=\{(x, y): \varphi(x)=\theta(y)\}$. If $N$ is a subset of $X \times Y, N_{x}=N(x)=\{y \in Y:(x, y) \in N\}$ is a set such that $\{x\} \times N_{x}=N \cap(\{x\} \times Y)$. For our purposes we will consider sets $N$ contained in $X \circ{ }^{Z} Y$ and thus $N_{x} \subseteq \theta^{-1}(\phi(x))$.

For the convenience of the reader we will now state some simplified results of [6] that we will be using. First we note that the assumption that $Y$ be point-transitive in [6] was not needed. 
Corollary 1.4 of [6]. Suppose $X$ is point-transitive, $\varphi: X \rightarrow Z$, $\theta: Y \rightarrow Z$ are surjective homomorphisms, and $\theta$ has $a \mathrm{RIM}, \lambda$. If $x_{0} \in X_{m}$, $A$ a Borel set contained in $\theta^{-1}(\phi)\left(x_{0}\right)$, and $N=\operatorname{cls}\left(\left(\left\{x_{0}\right\} \times A\right) T\right)$, then for $x \in S(\phi)\left(x_{0}\right), \lambda_{\phi\left(x_{0}\right)}\left(A \cap N_{x}\right)=\lambda_{\phi\left(x_{0}\right)}(A)$ (that is, $\left.\lambda_{\phi\left(x_{0}\right)}\left(A \backslash N_{x}\right)=0\right)$. If in addition $A=B \cap C$ where $B$ is a Borel set contained in the support of $\lambda_{\phi\left(x_{0}\right)}$ with $\lambda_{\phi\left(x_{0}\right)}(B)=1$ and $C$ is a non-empty open set, then $A \subseteq N_{x}$.

Compare this with Lemma 2.6 below.

TheOREM 1.5 of [6]. Suppose $X$ is minimal and $Q: X \rightarrow Z$ has a RIM, $\lambda$. Then for $x$ in the support of $\lambda_{\phi(x)}, Q(\phi)(x)=S(\phi)(x)$.

1. A main consequence of this section is that if $\phi:(X, T) \rightarrow(Z, T)$ has a RIM, $\lambda$, then for some $z$ in $Z$ there exist $x_{1}, x_{2} \in \phi^{-1}(z)$ such that $o c\left(x_{1}, x_{2}\right) \supseteq Q(\phi)\left(x_{1}\right) \times Q(\phi)\left(x_{2}\right)$. This holds for all $z$ that satisfy three types of conditions, $z \in Z_{1} \cap Z_{2} \cap Z_{3}$, where $z_{1}, z_{2}, z_{3}$ are as follows.

First consider $\pi: X \rightarrow X / S(\phi)$, with $X$ metric. Then by Lemma 3.1 of [9] there exist a residual subset $X_{1}$ of $X$ such that $\pi$ is open at each $x$ in $X_{1}$. By Proposition 3.1 of [10] the set $Z_{1}=\left\{z \in Z: X_{1} \cap \phi^{-1}(z)\right.$ is residual in $\left.\phi^{-1}(z)\right\}$ is residual in $Z$. Note for $x \in X_{1}$, every neighborhood $V$ of $x$ has $\pi(V)$ a neighborhood of $\pi(x)$ and $V^{\prime}=V \cap \pi^{-1}(\operatorname{int}(\pi V))$ a neighborhood of $x$ with $\pi^{-1}\left(\pi\left(V^{\prime}\right)\right)=\pi^{-1}(\operatorname{int}(\pi V))$ open, that is $S(\phi)\left(V^{\prime}\right)=$ $\pi^{-1}\left(\pi\left(V^{\prime}\right)\right)$ open. As noted in [10], $V \subseteq \operatorname{cls}\left(V^{\prime}\right)$.

More importantly, for fixed $z \in Z_{1}$ every open set $V^{*}$ in $\phi^{-1}(z)$ contains an open set $V^{* \prime}$ in $\phi^{-1}(z)$ such that $S(\phi)\left(V^{* \prime}\right)$ is open - indeed $V^{*}=V \cap \phi^{-1}(z)$ where $V$ is open, and $V^{*} \cap X_{1} \neq \varnothing$ and so there is an open set $V^{\prime}$ such that $V^{\prime} \cap \phi^{-1}(z) \neq \varnothing$ and $S(\phi)\left(V^{\prime}\right)$ is open, thus $V^{* \prime}=V^{\prime} \cap \phi^{-1}(z)$ has $S(\phi)\left(V^{* \prime}\right)=S(\phi)\left(V^{\prime} \cap \phi^{-1}(z)\right)=S(\phi)\left(V^{\prime}\right) \cap$ $\phi^{-1}(z)$ open in $\phi^{-1}(z)$. Also $V \subseteq \operatorname{cls}\left(V^{\prime}\right)$, so $V^{*}=V \cap \phi^{-1}(z) \subseteq\left(\operatorname{cls} V^{\prime}\right)$ $\cap \phi^{-1}(z)$.

REMARK. Note that in the non-metric case if $Y$ is a singleton we have that every open set $V$ contains an open set $V^{*}$ such that $S\left(V^{*}\right)$ is open, $V \subseteq \operatorname{cls}\left(V^{*}\right)$, and $A \cap V \subseteq V^{*}$ where $A=\{x: \phi: X \rightarrow X / S$ is open at $x\}$. To prove this consider $\phi: X \rightarrow X / S$. Then $\phi(V)$ has non-empty interior $W$. Take $V^{*}=V \cap \phi^{-1}(W)$. Then $S\left(V^{*}\right)=\phi^{-1}\left(\phi\left(V \cap \phi^{-1}(W)\right)\right)=$ $\phi^{-1}(W)$. Let $x \in V$, then for any neighborhood $U$ of $x, U \cap V \neq 0$ and $\phi(U \cap V)$ has non-empty interior. So $\phi(U \cap V) \subseteq W$ and $U \cap V \subseteq$ $\phi^{-1}(W)$. Thus $V \subseteq \operatorname{cls}\left(V \cap \phi^{-1}(W)\right)=\operatorname{cls}\left(V^{*}\right)$.

Recall that given a function $f$ from a metric space $X$ onto a metric space $Z$, if $f$ is a Borel map (in particular, when it is either upper semi-continuous or lower semi-continuous), then $f$ is continuous at a residual subset of $X$. 
Define $\Phi: X \rightarrow 2^{X}$ by $\Phi(x)=S(\phi)(x)$. Then $\Phi$ is upper semi-continuous. There exists a residual set $X_{2}$ of $X$ such that $\Phi$ is continuous at each $x \in X_{2}$. Then there exists a residual set $Z_{2}$ of $Z$ such that $X_{2} \cap \phi^{-1}(z)$ is residual in $\phi^{-1}(z)$ for $z \in Z_{2}$.

Finally, if $\phi: X \rightarrow Z$ admit a relative invariant measure (RIM), $\lambda$, then the function $g: Z \rightarrow 2^{X}$ defined by $g(z)=$ support of $\lambda z$ is lower semicontinous. Then one can show that there exists a residual set $Z_{3}$ of $Z$ such that the support of $\lambda z$ equals $\Phi^{-1}(z)$ for $z$ in $Z_{3}$ (Proposition 3.3 of [4]).

1.1. Proposition. Suppose for $i=1,2, \ldots, n \phi_{i}: X_{i} \rightarrow Z$ has a relative invariant measure $\lambda_{i}, z \in Z$ with $\phi_{i}^{-1}(z)=$ support of $\lambda_{i z}$, and $V_{i}$ are open subsets of $\phi_{i}^{-1}(z)$ with $Q\left(\phi_{i}\right)\left(V_{i}\right)$ open in $\phi_{i}^{-1}(z)$. Then

$$
\begin{aligned}
N & =o c\left(V_{1} \times \cdots \times V_{n}\right) \supseteq Q\left(\phi_{1}\right)\left(V_{1}\right) \times \cdots \times Q\left(\phi_{n}\right)\left(V_{n}\right) \\
& =S\left(\phi_{1}\right)\left(V_{1}\right) \times \cdots \times S\left(\phi_{n}\right)\left(V_{n}\right) .
\end{aligned}
$$

Proof. The last equality follows from 1.5 of [6].

Now $N \supseteq Q\left(\phi_{1}\right)\left(V_{1}\right) \times V_{2} \times \cdots \times V_{n}$ by Corollary 1.4 of [6] since $V_{2} \times \cdots \times V_{n}$ is open and $X_{2} \times \cdots \times X_{n}$ has a relative invariant measure. So $N \supseteq Q\left(V_{1}\right) \times Q\left(V_{2}\right) \times V_{3} \times \cdots \times V_{n}$ by 1.4 of [6] since $Q\left(V_{1}\right) \times$ $V_{3} \times \cdots \times V_{n}$ is open, the proposition follows by induction.

1.2. Proposition. For $i=0,1, \ldots, n$. Suppose $\left(X_{i}, T\right)$ is a minimal flow with $T$ abelian. Then for any $x_{0}$ in $X_{0}$ and for any open sets $V_{i}$ in $X_{i}$, $i=1,2, \ldots, n$ there exist open sets $U_{0}, U_{1}, \ldots, U_{n}$ such that $x_{0} \in \operatorname{cls}\left(U_{0}\right)$; $U_{i} \subseteq V_{i}, i=1,2, \ldots, n$; and

$$
\begin{aligned}
& o c\left(\left\{x_{0}\right\} \times V_{1} \times \cdots \times V_{n}\right) \\
& \quad \supseteq o c\left(U_{0} \times U_{1} \times \cdots \times U_{n}\right) \supseteq \operatorname{cls}\left[Q\left(U_{0}\right) \times Q\left(U_{1}\right) \times \cdots \times Q\left(U_{n}\right)\right] \\
& \quad \supseteq\left\{x_{0}\right\} \times Q\left(U_{1}\right) \times \cdots \times Q\left(U_{n}\right) .
\end{aligned}
$$

If $X_{0} \rightarrow X_{0} / Q$ is open at $x_{0}$, then we can take $U_{0}$ with $x_{0} \in U_{0}$.

Proof. Let $V\left(t_{1}, t_{2}, \ldots, t_{n}\right)$ denote $V_{1} t_{1} \times V_{2} t_{2} \times \cdots \times V_{n} t_{n}$ where $t_{i} \in$ $T$. Then there exist finite many $n$-tuples $s_{1}, s_{2}, \ldots, s_{m}$ in $\Pi_{1}^{n} T$ such that $\cup V\left(s_{i}\right)=\Pi_{1}^{n} X_{i}$. Let $Y=\Pi_{1}^{n} X_{i}$. Then $\cup_{1}^{n}\left[\left\{x_{0}\right\} \times V\left(s_{i}\right)\right]=\left\{x_{0}\right\} \times Y$. So $\cup_{1}^{n}\left(\left[\left\{x_{0}\right\} \times V\left(s_{i}\right)\right] t\right)=\left(\cup\left[\left\{x_{0}\right\} \times V\left(s_{i}\right)\right]\right) t=\left(\left\{x_{0}\right\} \times Y\right) t=$ $\left\{x_{0} t\right\} \times Y$. Therefore $\cup_{1}^{n} \operatorname{cls}\left(\left[\left\{x_{0}\right\} \times V\left(s_{i}\right)\right] T\right)=X_{0} \times Y$ and thus $\operatorname{cls}\left(\left[\left\{x_{0}\right\} \times V\left(s_{i}\right)\right] T\right)$ has non-empty interior, $I$, for some $i$. Then for some $t$ in $T,\left[\left\{x_{0}\right\} \times V\left(s_{i}\right)\right] t \cap I \neq \varnothing$. So there exist open sets $U_{0}^{\prime}$, $U_{1}^{\prime}, \ldots, U_{n}^{\prime}$ with $x_{0} \in U_{0}^{\prime}$, and $U_{1}^{\prime} \times \cdots \times U_{n}^{\prime} \subseteq V\left(s_{i}\right)$ such that $\left(U_{0}^{\prime} \times U_{1}^{\prime}\right.$ $\left.\times \cdots \times U_{n}^{\prime}\right) t \subseteq I$ and so $U_{0}^{\prime} \times U_{1}^{\prime} \times \cdots \times U_{n}^{\prime} \subseteq \operatorname{cls}\left(\left[\left\{x_{0}\right\} \times V\left(s_{i}\right)\right] T\right)$. 
Let $s_{i}=\left(t_{1}, \ldots, t_{n}\right)$, then since $T$ is abelian,

$$
U_{0}^{\prime} \times U_{1}^{\prime} t_{1}^{-1} \times \cdots \times U_{n}^{\prime} t_{n}^{-1} \subseteq \operatorname{cls}\left(\left[\left\{x_{0}\right\} \times V_{1} \times \cdots \times V_{n}\right] T\right) .
$$

Then by the remarks above there exist open sets $U_{i} \subseteq U_{i}^{\prime} t_{i}^{-1}$ such that $Q\left(U_{i}\right)$ is open $i=0,1, \ldots, n$, and $x_{0} \in U_{0}^{\prime} \subseteq \operatorname{cls} U_{0}$. When $X_{0} \rightarrow X_{0} / Q$ is open at $x_{0}$ we have $x_{0} \in U_{0}$. Then the proposition follows by 1.1.

1.3. Theorem. Suppose for $i=1,2, \ldots, \varphi_{i}: X_{i} \rightarrow Z$ has $a \mathrm{RIM}, \lambda_{i}$, and $X_{i}$ is a metric minimal flow. Let $z_{0} \in \cap_{i=1}^{\infty}\left(Z_{1}^{i} \cap Z_{2}^{i} \cap Z_{3}^{i}\right),\left(Z_{j}^{i}=Z_{j}\right.$ as above for $\left.\phi_{j}\right)$. Given $x_{i}$ in $X_{0}^{i}=\phi_{i}^{-1}\left(z_{0}\right)$ and dense $G_{\delta}$ subsets $G^{i}$ of $X_{0}^{i}$ there exist points $x_{i}^{\prime}$ in $G^{i}$ such that $x_{i}^{\prime} \in B\left(x_{i}, 1 / i\right)$ and oc $\left(x_{1}^{\prime}, x_{2}^{\prime}, \ldots\right) \supseteq$ $Q\left(\phi_{1}\right)\left(x_{1}^{\prime}\right) \times Q\left(\phi_{2}\right)\left(x_{2}^{\prime}\right) \times \cdots . \quad($ Recall $Q(\phi)(x)=S(\phi)(x)$ for $x \in$ $\phi^{-1}\left(Z_{3}\right)$.)

Proof. Let $B\left(y_{1}, \ldots, y_{n} ; \varepsilon\right)$ denote $B\left(y_{1}, \varepsilon\right) \times \cdots \times B\left(y_{n}, \varepsilon\right)$. Fix $\varepsilon>0$. Consider any set $\left\{x_{i}\right\}, x_{i} \in X_{0}^{i} \cap X_{2}^{i}$. For each $i$ use the continuity of $\Phi_{i}$ at $x_{i}$ to associate a neighborhood $U_{i}^{*}=U_{i}^{*}\left(x_{i}, \varepsilon\right)$ of $x_{i}$ with $x_{i}$ and $\varepsilon$ such that if $x \in U_{i}^{*}$ and $y_{i} \in Q\left(\phi_{i}\right)\left(x_{i}\right)$, then $Q\left(\phi_{i}\right)(x) \cap B\left(y_{i}, \varepsilon\right) \neq \varnothing$; and if $\varepsilon^{\prime}>\varepsilon$, then $U_{i}^{*}\left(x_{i}, \varepsilon^{*}\right) \supseteq U_{i}^{*}\left(x_{i}, \varepsilon\right)$. Let $U_{i}=U_{i}\left(x_{i}, \varepsilon\right)=U_{i}^{*} \cap X_{0}^{i}$ and $y_{i} \in Q\left(\phi_{i}\right)\left(x_{i}\right)$; note $x_{i} \in U_{i}$. Now for each $n$ consider the set $W_{n}=$ $W\left(y_{1}, \ldots, y_{n} ; \varepsilon\right)=\left\{w \in U_{1} \times \cdots \times U_{n}: w t \in B\left(y_{1}, \ldots, y_{n} ; \varepsilon\right)\right.$ for some $t$ in $T$ \}. Clearly $W_{n}$ is open (in $X_{0}^{1} \times \cdots \times X_{0}^{n}$ ). Also $W_{n}$ is a dense subset of $U_{1} \times \cdots \times U_{n}$; since for any basic open subset $V=V_{1} \times \cdots \times V_{n}$ of $U_{1} \times \cdots \times U_{n}$ in $X_{0}^{1} \times \cdots \times X_{0}^{n}$ take an open subset $V^{*}=V_{1}^{*} \times \cdots \times V_{n}^{*}$ with $Q\left(\phi_{i}\right)\left(V_{i}^{*}\right)$ open. Then for any point $\left(x_{1}^{*}, \ldots, x_{n}^{*}\right)$ in $V^{*}$, there exists $y_{i}^{*} \in Q\left(\phi_{i}\right)\left(x_{i}^{*}\right) \cap B\left(y_{i}, \varepsilon\right)$ for $i=1, \ldots, n$, and by 1.1 , we have $o c\left(V^{*}\right) \supseteq$ $Q\left(\phi_{1}\right)\left(V_{1}^{*}\right) \times \cdots \times Q\left(\phi_{n}\right) \ni\left(y_{1}^{*}, \ldots, y_{n}^{*}\right)$; so there exists $t$ in $T$ with $V^{*} t \cap B\left(y_{1}, \ldots, y_{n} ; \varepsilon\right) \neq 0$, and thus $W_{n}$ is dense.

Consider a cover of $Q\left(\phi_{1}\right)\left(x_{1}\right) \times \cdots \times Q\left(\phi_{n}\right)\left(x_{n}\right)$ by sets of the form $B\left(y_{1}, \ldots, y_{n} ; \varepsilon\right)$ where $y_{i} \in Q\left(\phi_{i}\right)\left(x_{i}\right)$. Take a finite subcover and the (finite) intersection $B_{n}$ of the corresponding $W_{n}$ 's, then $B_{n}$ is open (in $\left.X_{0}^{1} \times \cdots \times X_{0}^{n}\right)$ and is dense in $U_{1} \times \cdots \times U_{n}$. By continuity, for each $b$ in $B_{n}$, there is a neighborhood $E(b)$ of $b$ contained in $B_{n}$ such that any given open set in the finite subcover contains $E(b) t$ for some $t$ in $T$. From this it is clear that for any $\left(y_{1}, \ldots, y_{n}\right)$ in $Q\left(\phi_{1}\right)\left(x_{1}\right) \times \cdots \times Q\left(\phi_{n}\right)\left(x_{n}\right)$, $E(b) t \subseteq B\left(y_{1}, \ldots, y_{n} ; 2 \varepsilon\right)$ for some $t$ in $T$.

Now consider a given collection $x_{i} \in X_{0}^{i}, i=1,2, \ldots$ We may assume $x_{i} \in X_{0}^{i} \cap X_{2}^{i}$. Let $H_{j}^{i}, i, j=1,2, \ldots$, be dense open subsets of $X_{0}^{i}$ such that $H_{j+1}^{i} \subseteq H_{j}^{i}$ and $\cap_{j=1}^{\infty} H_{j}^{i}=X_{2}^{i} \cap X_{0}^{i} \cap G^{i}$ for $i=1,2, \ldots$ Start an induction with $x_{1}, x_{2}, n=2$ and $\varepsilon=\frac{1}{2}$. Take $B_{2}$ as above and $b_{2} \in\left(X_{2}^{1} \times\right.$ $\left.X_{2}^{2}\right) \cap\left(G^{1} \times G^{2}\right) \cap B_{2} \cap\left[B\left(x_{1}, 1\right) \times B\left(x_{2}, \frac{1}{2}\right)\right]$. Let $E_{2}=\left(H_{1}^{1} \times H_{2}^{2}\right) \cap$ $E\left(b_{2}\right) \cap B\left(b_{2}, \frac{1}{2}\right) \cap\left[B\left(x_{1}, 1\right) \times B\left(x_{2}, \frac{1}{2}\right)\right]$; note it is a neighborhood of $b_{2}$ in $X_{0}^{1} \times X_{0}^{2}$. Now consider $b_{2} \times\left\{x_{3}\right\}, n=3, \varepsilon=\frac{1}{3}$, and take $B_{3}$ as above 
and $b_{3} \in\left(X_{2}^{1} \times X_{2}^{2} \times X_{2}^{3}\right) \cap\left(G^{1} \times G^{2} \times G^{3}\right) \cap B_{3} \cap\left[E_{2} \times B\left(x_{3}, \frac{1}{3}\right)\right]$. Take a neighborhood $E_{3}$ of $b_{3}$ with $\operatorname{cls}\left(E_{3}\right) \subseteq\left(H_{2}^{1} \times H_{2}^{2} \times H_{2}^{3}\right) \cap E\left(b_{3}\right)$ $\cap B\left(b_{3}, \frac{1}{3}\right) \cap\left[E_{2} \times B\left(x_{3}, \frac{1}{3}\right)\right]$. Consider $b_{3} \times\left\{x_{4}\right\}, n=4, \varepsilon=\frac{1}{4}$, take $B_{4}$ as above and $b_{4} \in\left(X_{2}^{1} \times X_{2}^{2} \times X_{2}^{3} \times X_{2}^{4}\right) \cap\left(G^{1} \times G^{2} \times G^{3} \times G^{4}\right) \cap$ $\left[E_{3} \times B\left(x_{4}, \frac{1}{4}\right)\right] \cap B_{4}$. Continue in this way.

Note $\bigcap_{n=\mathrm{b} 2}^{\infty}\left(E_{n} \times \Pi_{n+1}^{\infty} X_{i}\right)$ is a singleton, say $\left\{\left(x_{1}^{\prime}, x_{2}^{\prime}, \ldots\right)\right\}$, and note $\left(x_{1}^{\prime}, x_{2}^{\prime}, \ldots\right) \in\left[\left(X_{2}^{1} \cap X_{0}^{1} \cap G^{1}\right) \times\left(X_{2}^{2} \cap X_{0}^{2} \cap G^{2}\right) \times\left(X_{2}^{3} \cap X_{0}^{3} \cap\right.\right.$ $\left.\left.G^{3}\right) \times \cdots\right] \cap\left[B_{1}\left(x_{1}, 1\right) \times B\left(x_{2}, \frac{1}{2}\right) \times B\left(x_{3}, \frac{1}{3}\right) \times \cdots\right]$. We claim $o c\left(x_{1}^{\prime}, x_{2}^{\prime}, \ldots\right) \supseteq Q\left(\phi_{1}\right)\left(x_{1}^{\prime}\right) \times Q\left(\phi_{2}\right)\left(x_{2}^{\prime}\right) \times \cdots$. For any $\left(y_{1}, y_{2}, \ldots\right)$ in $Q\left(\phi_{1}\right)\left(x_{1}^{\prime}\right) \times Q\left(\phi_{2}^{\prime}\right)\left(x_{2}^{\prime}\right) \times \ldots$, a basic neighborhood of it is of the form $B\left(y_{1}, \ldots, y_{n} ; \lambda\right) \times \prod_{n+1}^{\infty} X$ for some $n$ and $\lambda>0$. Let $U_{i}^{\prime}=U\left(x_{i}^{\prime}, \lambda\right)$ for $i=1,2, \ldots, n$. Take $j$ such that $b_{j} \in U_{1}^{\prime} \times \cdots \times U_{n}^{\prime} \times \Pi_{n+1}^{j} X$ and $1 /(j+1)<\lambda$. Then

$$
\left[Q\left(\phi_{1}\right)\left(b_{j 1}\right) \times \cdots \times Q\left(\phi_{n}\right)\left(b_{j n}\right)\right] \cap B\left(y_{1}, \ldots, y_{n} ; \lambda\right) \neq \varnothing,
$$

where $b_{j}=\left(b_{j 1}, \ldots, b_{j n}\right)$, (since $\left.b_{j i} \in U_{i}^{\prime}\right)$. Let $\left(y_{1}^{*}, \ldots, y_{n}^{*}\right)$ be a point in this intersection. Then there exists $t$ in $T$ such that $\left(x_{1}^{\prime}, x_{2}^{\prime}, \ldots\right) t \in E_{j+1} t \subseteq$ $B\left(y_{1}^{*}, \ldots, y_{n}^{*} ; 2 /(j+1)\right) \subseteq B\left(y_{1}, \ldots, y_{n} ; 3 \lambda\right)$. Thus $\left(y_{1}, y_{2}, \ldots\right) \in$ $o c\left(x_{1}^{\prime}, x_{2}^{\prime}, \ldots\right)$.

1.4. Corollary. Suppose $X$ is metric, minimal flow and $\phi: X \rightarrow Z$ has $a$ RIM. Then there exists $\left(x_{0}, x_{1}\right) \in X \times X$ such that $\phi^{\prime}: Y=X / R\left(x_{0}, x_{1}\right)$ $\rightarrow Z$ is an almost automorphic extension of $Z$ (i.e., there is a point $y$ in $Y$ with $\left.Q\left(\phi^{\prime}\right)(y)=\{y\}\right)$ where $R\left(x_{0}, x_{1}\right)$ is the smallest closed invariant equivalence relation containing $\left(x_{0}, x_{1}\right)$.

Proof. This is clearly the case if we take $\left(x_{0}, x_{1}\right)$ such that $o c\left(x_{0}, x_{1}\right)$ $\supseteq Q(\phi)\left(x_{0}\right) \times Q(\phi)\left(x_{1}\right)$.

2. In this section we develop some connections of a RIM on $\phi$ : $X \rightarrow Y$ to the relativized equicontinuous structure relation, $S(\phi)$, and apply them to study the orbit closures of sets of the form $\{x\} \times A^{2}$ $\times \cdots \times A^{n}$ in a product space and to give a special characterization $S(\phi)$ in the case when $(R(\phi), T)$ has a dense set of almost periodic points.

Suppose $\phi: X \rightarrow Y$ has a $\operatorname{RIM}, \lambda, X$ is minimal and $N$ is a closed invariant set in $R(\phi)$. Then $\phi_{N}: R(\phi) \rightarrow[0,1]$ defined by $\phi_{N}\left(x, x^{\prime}\right)=$ $\lambda_{\phi(x)}\left(N(x) \Delta N\left(x^{\prime}\right)\right)=2 \lambda_{\phi(x)}\left(N(x) \backslash N\left(x^{\prime}\right)\right)$ is continuous, [6] where $\{x\} \times N(x)=N \cap(\{x\} \times X)$ and $\Delta$ is the symmetric difference. So for each $N, \phi_{N}\left(x, x^{\prime}\right)$ is a pseudo-metric on each fiber that is invariant, $\phi_{N}\left(x t, x^{\prime} t\right)=\phi_{N}\left(x, x^{\prime}\right)$. Defining $R_{N}=\left\{\left(x, x^{\prime}\right) \in R(\phi): \phi_{N}\left(x, x^{\prime}\right)=0\right\}$, we have $X \rightarrow X / R_{N} \stackrel{\psi_{N}}{\rightarrow} Y$ and $\psi_{N}$ is an isometric homomorphism (and thus almost periodic). 
Consider $S^{*}(\phi)=\left\{\left(x, x^{\prime}\right): \phi_{N}\left(x, x^{\prime}\right)=0\right.$ for all closed invariant subsets $N$ of $R(\phi)\}$. Then by 1.2 of [6] $S(\phi) \subseteq S^{*}(\phi)$. We wish to show that $S^{*}(\phi) \subseteq S(\phi)$. Note by 1.2 of [6] $S^{*}(\phi)$ is closed and invariant. Suppose $\left(x, x^{\prime}\right) \in S^{*}(\phi)$. Let $\phi(x)=z_{0}$, let $x_{1} \in S_{\lambda}=$ support of $\lambda_{z_{0}}$, and let $p u \in M$ such that $x p u=x_{1}$. Note $\left(x p u, x^{\prime} p u\right) \in S^{*}(\phi)$. For any $V \in \Re_{x_{1}}$ consider $N=o c\left(\left\{x^{\prime} p u\right\} \times V \cap S_{\lambda}\right)$. By 1.4 of [6] $N \supseteq\left\{x_{1}\right\} \times$ $\left(V \cap S_{\lambda}\right)$, so $N \cap V \times\left(V \cap S_{\lambda}\right) \neq \varnothing$, so there exists $t_{V}$ in $T$ and $x_{V}$ in $V \cap S_{\lambda}$, such that $x_{V} t_{V} \in V$ and $x^{\prime} p^{\prime} t_{V} \in V$. Thus $x^{\prime}=x p u \in$ $Q(\phi)\left(x^{\prime} p u\right)$ and so $\left(x u, x^{\prime} u\right) \in Q(\phi)$ and $\left(x, x^{\prime}\right) \in S(\phi)$. Thus we have the following proposition.

2.1. Proposition. If $\phi: X \rightarrow Z$ has a RIM, $\lambda$, then $\left\{\left(x, x^{\prime}\right) \in R(\phi)\right.$ : $\lambda_{\phi(x)}\left(N(x) \Delta N\left(x^{\prime}\right)\right)=0$ for all closed invariant sets $N$ in $\left.R(\phi)\right\}=S(\phi)=$ $\left\{\left(x, x^{\prime}\right) \in R(\phi):\left(x u, x^{\prime} u\right) \in Q(\phi)\right.$ for some (and thus every) $\left.u \in J\right\}$.

2.2. Proposition. Suppose $\Phi: X \rightarrow Y$ has $a$ RIM, $\lambda$, and $X$ and $Y$ are minimal. If $\phi$ is open and $S(\phi)=R(\phi)$, then $Q(\phi)=S(\phi)$.

Proof. Let $\left(x, x^{\prime}\right) \in R(\phi)=S(\phi)$, we will $\operatorname{show}\left(x, x^{\prime}\right) \in \overline{Q(\phi)}=$ $Q(\phi)$. Let $U$ and $V$ be open neighborhoods of $x$ and $x^{\prime}$ respectively. Let $x_{0}$ be any point in the support of $\lambda_{\phi(x)}$. Since $\phi$ is an open map, $\phi(V) \cap \phi(U)$ is an open neighborhood of $\phi(x)$. There exist $t_{0}$ in $T$ with $x_{0} t_{0} \in V$ and $\phi\left(x_{0} t_{0}\right) \in \phi(V) \cap \phi(U)$. So there is $x_{1} \subset U$ with $\phi\left(x_{1}\right)=$ $\phi\left(x_{0} t_{0}\right)$; then $\left(x_{1} t_{0}^{-1}, x_{0}\right) \in R(\phi)=S(\phi)$ and by 1.5 of [6], $x_{1} t_{0}^{-1} \in$ $S(\phi)\left(x_{0}\right)=Q(\phi)\left(x_{0}\right)$. Therefore $\left(x_{1}, x_{0} t\right)=\left(x_{1} t_{0}^{-1}, x_{0}\right) t_{0} \in Q(\phi)$ and $\left(x, x^{\prime}\right) \in \overline{Q(\phi)}=Q(\phi)$.

2.3. Lemma. Given $\phi: X \rightarrow Y, \theta: Y \rightarrow Z, X$ minimal. Let $x \in X$ and $y=\phi(x)$. Then for any $y^{\prime} \in S(\theta)(y)$ there exists $x^{\prime} \in S(\theta \circ \phi)(x)$ with $y^{\prime}=\phi\left(x^{\prime}\right)$. (Note this is somewhat stronger than the statement $\phi \times$ $\phi(S(\theta \circ \phi))=S(\theta)$.

Proof. By 14.2 of $\left[\mathbf{2}_{\mathbf{b}}\right], \phi \times \phi(Q(\theta \circ \phi))=Q(\theta)$. Consider $M \stackrel{\psi}{\rightarrow} X$ with $\psi(m)=x m$. Then $\phi \times \phi(\psi \times \psi(Q(\theta \circ \phi \circ \psi)))=Q(\theta)$. Let $u \in J$ with $x u=x$. Note $Q(\theta \circ \phi \circ \psi)$ is left invariant under $G=M_{0} u, M_{0}=$ $(\theta \circ \phi \circ \psi)^{-1}(y)$; and so $S(\theta \circ \phi \circ \psi)$ is also, since $g \times g(S(\theta \circ \phi \circ \psi))$ is a closed invariant equivalence relation containing $g \times g(Q(\theta \circ \phi \circ \psi))=$ $Q(\theta \circ \phi \circ \psi)$, for $g \in G$. Let $R$ denote $\phi \times \phi(\psi \times \psi(S(\theta \circ \phi \circ \psi)))$. Also $S(\theta) \supseteq \phi \times \phi(S(\theta \circ \phi)) \supseteq R$. To show the reverse inclusion first note $Q(\theta)=\phi \times \phi(\psi \times \psi(Q(\theta \circ \phi \circ \psi))) \subseteq R$. Also $R$ is closed and invariant; we will now show that $R$ is an equivalence relation and thus $S(\theta) \subseteq R$ and the lemma will follow. We only need to show that if $\left(y_{1}, y_{2}\right) \in R$ and 
$\left(y_{2}, y_{3}\right) \in R$, then $\left(y_{1}, y_{3}\right) \in R$. Let $m_{1}, m_{2}, m_{2}^{\prime}, m_{3} \in M$ with $\left(m_{1}, m_{2}\right)$, $\left(m_{2}^{\prime}, m_{3}\right) \in S(\theta \circ \phi \circ \psi)$ and $\phi \circ \psi\left(m_{i}\right)=y_{l}, i=1,2,3 \phi \circ \psi\left(m_{2}^{\prime}\right)=y_{2}$. Choose $m \in M$ so that $m m_{2}^{\prime} \in m_{2} J$. Then $\left(m m_{2}^{\prime}, m m_{3}\right) \in S(\theta \circ \phi \circ \psi)$ and $\left(m_{2}, m m_{2}^{\prime}\right) \in S(\theta \circ \phi \circ \psi)$, and so $\left(m_{1}, m m_{3}\right) \in S(\theta \circ \phi \circ \psi)$. Also $\phi \circ \psi\left(m m_{3}\right)=y_{3}$ since $\phi \circ \psi\left(m m_{2}^{\prime}\right)=\phi \circ \psi\left(m_{2}^{\prime}\right)$; so $\left(y_{1}, y_{3}\right) \in R$. Thus we have that $S(\theta)=\phi \times \phi(S(\theta \circ \phi))=R$.

Now suppose $y^{\prime} \in S(\phi)(y)$, and $\left(m, m^{\prime}\right) \in S(\theta \circ \phi \circ \psi)$ with $\phi \circ \psi(m)=y, \phi \circ \psi\left(m^{\prime}\right)=y^{\prime}$. We may assume $m=m u$ since $S(\theta \circ \phi \circ \psi)$ is an equivalence relation. Then $\left(u, m^{-1} m^{\prime}\right) \in S(\theta \circ \phi \circ \psi)$ and $\psi(u)=x$. Let $x^{\prime}=\psi\left(m^{-1} m^{\prime}\right)$. Then $\left(x, x^{\prime}\right) \in S(\theta \circ \phi)$ and $\phi\left(x^{\prime}\right)=y^{\prime}$. Thus the lemma is proved.

2.4. LeMMA. Let $M$ be the universal minimal set, $Z$ a minimal flow, $z$ a fixed element of $Z, u \in J$ with $z u=z$, and $\psi: M \rightarrow Z$ be defined by $\psi(p)=z p, p \in M$.

If $p \in S(\psi)(u)$ and $p v=p, v \in J$, then $[S(\psi)(u)] v=[S(\psi)(u)] p$.

Proof. If $m \in S(\psi)(u)$, then $m p \in S(\psi)(u p)=S(\psi)(u)$ since $u p=p$ and $S(\psi)$ is a closed invariant equivalence relation. So $S(\psi)(u) p \subseteq$ $S(\psi)(u)$ and so $S(\psi)(u) p \subseteq S(\psi)(u) v$.

Let $p^{-1}$ be the inverse of $p$ in the group $M v$. Then $S(\psi)(u) p^{-1} \in$ $S(\psi)(u)$ and $S(\psi)(u) v=S(\psi)(u) p^{-1} p \subseteq S(\psi)(u) p$.

2.5. Corollary. Using the same notation as in Lemma 2.4 and $v \in J$; if $p \in S(\psi)(u), p v=p$, and $\phi: X \rightarrow Z$, then $S(\phi)(x) p=S(\phi)(x) v$ for all $x$ in $X$ with $\phi(x)=z$ and $x u=x$.

Proof. Straightforward.

The following lemma is a variation of Corollary 1.4 of [6].

2.6. Lemma. Suppose $\phi: X \rightarrow Z, \theta: Y \rightarrow Z, Z$ minimal and $\theta$ has a RIM (section), $\lambda$. Let $r \in X$ and $z=\phi(r)$, let $V$ be an open set in the support of $\lambda_{z}$, and let $N=o c(\{r\} \times V)$ and $v \in J$, with $z v=z$. Then $N \supseteq\{r v\} \times v$. (Note $X$ and $Y$ are not required to be minimal, otherwise it would be trivial in view of 1.4 of [6] since $r v$ and $r$ are proximal and so $(r v, r)$ would be in $S(\phi)$.)

Proof. We will assume the reader is familiar with the notation and definitions in [6]. Let $W \in \mathfrak{N}\left(N_{r v)}\right.$ with $\lambda_{z}(W)<\lambda_{z}\left(N_{r v}\right)+\varepsilon$. Then there exists $t$ in $T$ for which $N_{r} t \subseteq W$ and $N_{r v} t \subseteq W$ and $\left|\lambda_{z t}(W)-\lambda_{z}(W)\right|<\varepsilon$. 
Then

$$
\begin{aligned}
\lambda_{z}\left(N_{r} \backslash N_{r v}\right) & =\lambda_{z t}\left(N_{r} t \backslash N_{r v} t\right) \leq \lambda_{z t}\left(W \backslash N_{r v} t\right) \\
& =\lambda_{z t}(W)-\lambda_{z t}\left(N_{r v} t\right) \\
& =\lambda_{z t}(W)-\lambda_{z}\left(N_{r v}\right) \\
& \leq\left|\lambda_{z t}(W)-\lambda_{z}(W)\right|+\left|\lambda_{z}(W)-\lambda_{z}\left(N_{r v}\right)\right| \\
& <2 \varepsilon .
\end{aligned}
$$

Thus $\lambda_{z}\left(N_{r} \backslash N_{r v}\right)=0$. Now $\lambda_{z}\left(V \backslash N_{r v}\right) \leq \lambda\left(N_{r} \backslash N_{r v}\right)=0$, and so $V \backslash N_{r v}$ $=\varnothing$ since $V \backslash N_{r v}$ is open in the support of $\lambda_{z}$. Thus $V \subseteq N_{r v}$.

2.7 Lemma. Suppose $\phi: X \rightarrow Z, \theta: Y \rightarrow Z, Z$ minimal, and $\theta$ has a RIM. Let $x_{0} \in X, z_{0}=\phi\left(x_{0}\right)$, and let $\Lambda$ be a non-empty subset of the set $\{\lambda: \lambda$ is a RIM for $\theta\}$. Let $S_{\lambda}$ be the support of $\lambda_{z_{0}}$ and $S=\operatorname{cls}\left(\cup_{\lambda \in \Lambda} S_{\lambda}\right)$. Let $C$ be an open set in $\theta^{-1}\left(z_{0}\right)$ and $A=S \cap C$. Consider $N=$ $o c\left(\left\{x_{0}\right\} \times A\right)$. Then $N_{x} \supseteq A$ for all $x \in S_{m}(\phi)\left(x_{0}\right)$. Note if $X$ is minimal, $S_{m}(\phi)=S(\phi)$. In addition if $v \in J$ with $z_{0} v=z_{0}$, then $N \supseteq\left\{x_{0} v\right\} \times A$.

Proof. By 1.4 of [6], $A \cap S_{\lambda}=C \cap S_{\lambda} \subseteq N_{x}$ for every $\lambda$ in $\Lambda$. So $A \cap\left(\cup S_{\lambda}\right)=C \cap\left(\cup S_{\lambda}\right) \subseteq N_{x}$ and $\operatorname{cls}\left(C \cap\left(\cup S_{\lambda}\right)\right) \subseteq N_{x}$. If $y \in C \cap$ $S$, then for every open neighborhood $V$ of $y$ in $\theta^{-1}\left(z_{0}\right)$ with $V \subseteq C$, there exists $y_{\lambda}$ in $S_{\lambda}$ for some $\lambda$ in $\Lambda$ with $y_{\lambda} \in V \subseteq C$; thus $A=C \cap S \subseteq$ $\operatorname{cls}\left(C \cap\left(\cup S_{\lambda}\right)\right) \subset N_{X}$. The additional statement follows similarly from 2.6.

2.8. Proposition. Given homomorphisms $\alpha: W \rightarrow X, \phi: X \rightarrow Z$ where $\phi$ has $\operatorname{RIM} \lambda$ and $W$ is minimal, let $\psi=\phi \circ \alpha$. Then there exists a strongly proximal extension $\theta: Z^{\sim} \rightarrow Z$ such that the following diagram commutes

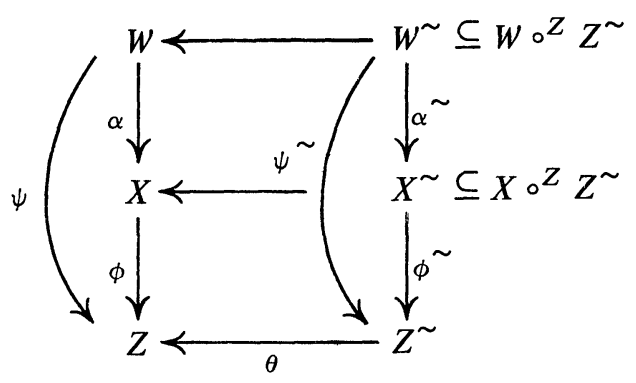

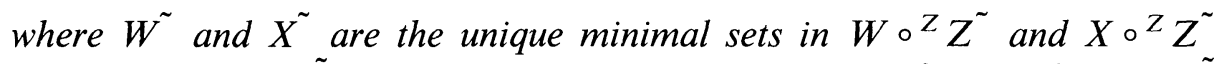
respectively. And $\psi_{\sim}^{\sim}$ has a $\operatorname{RIM} \gamma$, that induces the $\operatorname{RIM} \lambda_{\theta(\nu)} \times \delta_{\nu}, \nu \in Z^{\sim}$ on $\tilde{\phi}$. $\left(\delta \nu\right.$ is the point mass at $\left.\nu \in Z^{\tilde{\nu}}\right)$. 
REMARK. (a) Compare this with 5 of [7].

(b) When $W$ is the universal minimal set $M$, we have $M^{\tilde{T}}$ which is in fact isomorphic with $M$ through the $\operatorname{map}(p, \gamma) \rightarrow p$.

(c) If $Z$ is a universal strongly proximal flow, then $\theta: Z^{\sim} \rightarrow Z$ is an isomorphism and any RIM on $\phi$ can be lifted to a RIM on $\psi$.

Proof. We assume the reader is familiar with the contents of [4]. Let $x_{0} \in X_{0}, w_{0} \in W$ with $\alpha\left(w_{0}\right)=x_{0}$, and $u \in J$ with $w_{0} u=w_{0}$. Consider $\hat{\psi}: \mathfrak{R}(W) \rightarrow \Re(Z), \hat{\alpha}: \mathfrak{T}(W) \rightarrow \mathfrak{T}(X)$. Let $P=\overline{\operatorname{co}}\left(o c\left(\lambda_{z_{0}}\right)\right)$ and note $\hat{\phi}: P \rightarrow \mathfrak{R}(Z)$ is $\mathfrak{R}(Z)$ irreducible since $\lambda$ is a RIM. Let $Q$ be a $P$-irreducible subset of $\hat{\alpha}^{-1}(P)$ and note that $Q$ is also $\Re(Z)$ irreducible. Let $Z^{\tilde{}}=\overline{\operatorname{ex}}(Q)$ and $\theta=\hat{\psi} \mid Z^{\sim}$ - the restriction of $\hat{\psi}$ to $Z^{\tilde{z}}$; we identify $z$ in $Z$ with $\delta_{z}$ and consider $\theta: Z^{\sim} \rightarrow Z$. Let $X^{\sim}, W^{\sim}$ be the unique minimal sets in $\left\{(x, \nu) \in X \times Z^{\sim}: \phi(x)=\theta(\nu)\right\},\left\{(p, \nu) \in W \times Z_{\sim}^{\sim}: \phi \circ \alpha(p)=\right.$ $\theta(\nu)\}$ respectively. Let $\phi_{\sim}^{\sim}$ be the projection of $X^{\sim}$ onto $Z^{\sim}$ and $\psi^{\sim}$ be the projection of $W$ onto $Z^{\sim}$. For each $\nu$ in $Z^{\sim}$ the measure $\nu \times \delta_{\nu}$ on $W \times Z^{\sim}$ is supported in $\tilde{W^{\prime}}$ and the map $\gamma: \tilde{Z} \rightarrow \mathfrak{R}(\tilde{W}) \gamma_{\nu}=\nu \times \delta_{\nu}$ is a RIM

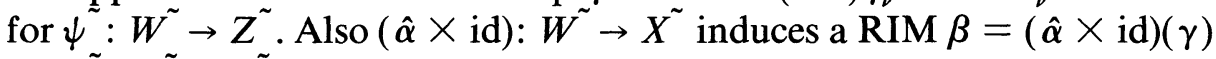
on $\tilde{\phi}: X^{\sim} \rightarrow Z^{\sim}$ by $\beta_{\nu}=(\hat{\alpha} \times$ id $)\left(\gamma_{\nu}\right)=\hat{\alpha}(\nu) \times \delta_{\nu}$; note $\hat{\alpha}\left(\nu_{0}\right)=\lambda_{z_{0}}$ for some $\nu_{0}$ in $Z^{\sim}$ and so $\theta\left(\nu_{0}\right)=z_{0}$ and $\beta_{\nu}=\lambda_{\theta(\nu)} \times \delta_{\nu}$ for $\nu$ in $Z^{\sim}$.

2.9. ThEOREM. Suppose for $i=1,2,3, \ldots, n, \phi_{i}: X^{i} \rightarrow Z$ are homomorphisms and $X^{i}$ is a minimal flow. Suppose $\phi_{1}$ has a RIM, $\lambda$. Let $z_{0} \in Z$, $X_{0}^{i}=\phi_{i}^{-1}\left(z_{0}\right)$. Then, there exist non-empty subsets $J^{*} \subseteq J^{\prime}$ of $J$ such that $X_{0}^{i} J^{*}, X_{0}^{i} J^{\prime}$ are compact subsets of $X_{0}^{i}$ and such that given $A^{i}=\left(X_{0}^{i} J^{*}\right) \cap V^{i}$ where $V^{i}$ is an open subset of $X_{0}^{i}, x, x^{\prime} \in X_{0}^{1} J^{\prime}$ with $\left(x, x^{\prime}\right) \in S\left(\phi_{1}\right)$, and $N=o c\left(\{x\} \times A^{2} \times \cdots \times A^{n}\right)$ we have $N \supseteq\left\{x^{\prime}\right\} \times A^{2} \times \cdots \times A^{n}$.

REMARK (a) If $u, v \in J^{\prime}, x_{1} u \in X_{0}^{1}, x_{2} v \in X_{0}^{2}$, and

$$
N=\operatorname{oc}\left(\left\{\left(x_{1} u, x_{2} v\right)\right\}\right) \times A^{3} \times \cdots \times A^{n} ;
$$

then $N \supseteq\left\{\left(x_{1} u, x_{2} u\right)\right\} \times A^{3} \times \cdots \times A^{n}$.

(b) $X_{0}^{i} J^{*} \supseteq \bigcup\left\{X_{0}^{i} u: u \in J\right.$ for which $x^{\prime} u \in S_{\mu}$ for some $x^{\prime}$ in $X_{0}^{i}$ and some RIM, $\mu$, for $\left.\phi_{i}\right\}$ where $S_{\mu}$ is the support of $\mu_{z_{0}}$.

(c) $J^{*}$ and $J$ depend on $Z$ but not on the $\phi_{i}$ 's.

(d) For $n=2$ compare this with 1.4 of [6], where $\phi_{2}$ has a RIM and $\phi_{1}$ is not required to have a RIM.

2.10. Corollary. If $X=X^{i}, i=1,2, x \in X_{0} J^{*}, x^{\prime} \in X_{0} J^{\prime}$, and $\left(x, x^{\prime}\right) \in S(\phi)$, then there exist $x_{n}$ in $X_{0} J^{*}$ and $t_{n}$ in $T$ with $x_{n} \rightarrow x$, $x_{n} t_{n} \rightarrow x, x^{\prime} t_{n} \rightarrow x$; in particular $\left(x, x^{\prime}\right) \in Q(\phi)$.

Proof of 2.9. Let $u \in J$ with $z_{0} u=z_{0}$. Define $\psi: M \rightarrow Z$ by $p \rightarrow z_{0} p$. Let $M_{0}=\psi^{-1}\left(z_{0}\right)$. Fix $x_{0}^{i} \in \phi_{i}^{-1}\left(z_{0}\right)=X_{0}^{i}$ with $x_{0}^{i} u=x_{0}^{i}$ and define $\alpha_{i}$ : $M \rightarrow X^{i}$ by $\alpha_{i}(p)=x_{0}^{i} p$. Note $\psi=\alpha_{i} \circ \phi_{i}$. By 4.1 of [4], there is a 
strongly proximal extension $Z^{\sim}$ of $Z, Z^{\sim}$ is minimal, $\theta: Z^{\sim} \rightarrow Z$ is strongly

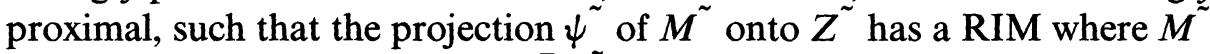
is the unique minimal set in $M \circ^{z} Z^{\sim}=\{(m, z) \in M \times Z: \psi(m)=\theta(z)\}$. By 2.7 we see that we are interested that the union of the supports of the RIM's on $\psi^{\sim}$ be as large as possible. We will now determine one aspect of the size of this union by "translating" measures. Given a RIM $\gamma$ on $\psi$ define the translation $p \gamma$ by $p \gamma_{\nu}(A)=\gamma_{\nu}(p A)$ for $p \in M_{0}=\psi^{-1}(\psi(u))$ and $\nu \in \tilde{Z}$. It is easy to see that $p \gamma$ is again a RIM on $\psi^{\tilde{\psi}}$. Let $\gamma_{0}=$ $\tilde{\psi}(u), \Gamma$ be the set of all RIM's on $\psi$, and $S_{\gamma}, \gamma \in \Gamma$, be the support of $\gamma_{\nu_{0}}$. From the above it is easy to see that $\operatorname{cls}\left\{\cup S_{\gamma}: \gamma \in \Gamma\right\}$ is of the form $\tilde{M}_{0}^{\tilde{J}} \tilde{J}^{*} \subseteq \tilde{M_{0}} \subseteq M_{0} \times\left\{\nu_{0}\right\}$ for some subset $J^{*}$ of $J$.

Now to prove the theorem we first show a similar result for $\tilde{\phi_{1}}$ and then reduce it to $\phi_{1}$. Suppose $A^{i}=\left(X_{0}^{i} J^{*}\right) \cap V^{i}$ where $V^{i}$ is open in $X^{i}$ and $\left(\left(x, \nu_{0}\right),\left(x^{\prime}, \nu_{0}\right)\right) \in S\left(\tilde{\phi_{1}}\right)$. Let $N^{2}=o c\left(\left\{x_{0}, \nu_{0}\right\}\right) \times\left(A^{2} \times\left\{\nu_{0}\right\}\right)$ $\times \cdots \times\left(A^{n} \times\left\{\nu_{0}\right\}\right)$. Then if $\left(p, \nu_{0}\right) \in \tilde{M_{0}}$ with $\alpha_{1}(p)=x$, there exist $\left(p^{\prime}, \nu_{0}\right) \in \tilde{M_{0}}$ with $\alpha_{1}\left(p^{\prime}\right)=x^{\prime}$ and $\left(\left(p, \nu_{0}\right),\left(p^{\prime}, \nu_{0}\right)\right) \in S\left(\psi^{\sim}\right)$. Consider

$$
\begin{aligned}
N^{*}=o c\left\{\left\{\left(p, \nu_{0}\right)\right\} \times\left(\alpha_{2}^{-1}\left(V^{2}\right)\right.\right. & \left.\times\left\{\nu_{0}\right\} \cap \tilde{M_{0}} J^{*}\right) \\
\times & \left.\cdots \times\left(\alpha_{n}^{-1}\left(V^{n}\right) \times\left\{\nu_{0}\right\} \cap \tilde{M_{0}} J^{*}\right)\right\} .
\end{aligned}
$$

For $i=2,3, \ldots, n$, let $\gamma_{i} \in \Gamma$, then $\Pi \gamma_{i}$ is a RIM and $S_{\pi \gamma_{i}}=\Pi S_{\gamma_{i}}$ so $\operatorname{cls}\left(\cup\left\{S_{\Pi \gamma_{i}}: \gamma_{i} \in \Gamma, i=2, \ldots, n\right\}\right)=\Pi_{2}^{n} M_{0} J^{*}$. So by 2.7

$$
\begin{aligned}
N^{*} \supseteq & \left\{\left(p^{\prime}, \nu_{0}\right)\right\} \times\left[\alpha_{2}^{-1}\left(V^{2}\right) \times\left\{\nu_{0}\right\} \cap \tilde{M_{0}} J^{*}\right] \\
& \times \cdots \times\left[a_{n}^{-1}\left(V^{n}\right) \times\left\{\nu_{0}\right\} \cap \tilde{M_{0}} J^{*}\right],
\end{aligned}
$$

and $N^{\sim} \supseteq\left\{\left(x^{\prime}, \nu_{0}\right)\right\} \times\left(A^{2} \times\left\{\nu_{0}\right\}\right) \times \cdots \times\left(A^{n} \times\left\{\nu_{0}\right\}\right)$, since if $\alpha_{i}\left(p_{i}\right)=$ $a_{i} \in A^{i}$ then $a_{i} u_{i}^{*}=a_{i}$ for some $u_{i}^{*} \in J^{*}$ and $\left(p_{i} u_{i}^{*}, \nu_{0}\right) \in\left(\alpha_{i}^{-1}\left(V^{i}\right) \times\right.$ $\left.\left\{\nu_{0}\right\}\right) \cap \tilde{M_{0}} J^{*}$. Thus

$$
N=o c\left(\{x\} \times A^{2} \times \cdots \times A^{n}\right) \supseteq\left\{x^{\prime}\right\} \times A^{2} \times \cdots \times A^{n} .
$$

We will now show that if $\left(x, x^{\prime}\right) \in S\left(\phi_{1}\right)$ and $\left(x, \nu_{0}\right),\left(x^{\prime}, \nu_{0}\right) \in X^{1^{\sim}}$, then $\left(\left(x, \nu_{0}\right),\left(x^{\prime}, \nu_{0}\right)\right) \in S\left(\phi_{1}\right)$, where $X^{1^{\sim}}$ is the unique minimal set in $X^{1} \circ^{z} Z^{2}$. (We let $J^{\prime}=\left\{v \in J: \nu_{0} v=\nu_{0}\right\}$ and note for $x \in X_{0}^{1},\left(x, \nu_{0}\right) \in$ $X^{\tilde{r}^{\sim}}$ iff $x \in X_{0}^{1} J^{\prime}$.) First suppose $x \in S_{\lambda}$, then there exist $x_{n}$ in $S_{\lambda}$ and $t_{n}$ in $T$ with $x_{n} \rightarrow x, x_{n} t_{n} \rightarrow x, x^{\prime} t_{n} \rightarrow x$ by 1.5 of [6]. Now $S_{\lambda} \times\left\{\nu_{0}\right\} \stackrel{\subset}{\sim} X^{\tilde{1}}$ since $\lambda_{\theta(\nu)} \times \delta_{\nu}, \nu \in Z^{\sim}$ is a RIM on $\phi_{1}^{\tilde{1}}$ by 5 of [7]. So $\left(x_{n}, \nu_{0}\right) \in X^{\tilde{\tau}}$ and we have $\left(\left(x, v_{0}\right),\left(x^{\prime}, v_{0}\right)\right) \in S\left(\tilde{\phi_{1}}\right)$. Now suppose $x \notin S_{\lambda}$, let $x_{1} \in S_{\lambda}$ and $w \in J^{*}$ such that $\left(x_{1}, \nu_{0}\right) w=\left(x_{1}, \nu_{0}\right)$. Let $p w \in M$ with $x p w=x_{1}$; then $\left(x^{\prime} p w, x p w\right) \in S\left(\phi_{1}\right)$ and $\left(\left(x^{\prime} p w, \nu_{0}\right),\left(x p w, v_{0}\right)\right) \in S\left(\phi_{1}\right)$. Multiplying on the right by $(p w)^{-1} \in M w$, we get $\left(\left(x^{\prime} w, \nu_{0}\right),\left(x w, \nu_{0}\right)\right) \in S\left(\tilde{\phi_{1}}\right)$ and therefore $\left(\left(x^{\prime}, \nu_{0}\right),\left(x, \nu_{0}\right)\right) \in S\left(\phi_{1}^{\tilde{l}}\right)$. Remark (a) is easily proved as above applying Lemma 2.7 to $\phi^{*}: o c\left(x_{1} u, x_{2} v\right) \rightarrow Z$. Remark (b) follows from 5 to [7] and 2.8 . 
2.11. Theorem. Suppose for $i=1,2, \ldots, n$ that $\phi_{i}: X_{i} \rightarrow Z$ has a RIM, $\mu_{i}$, and $X_{i}$ is minimal flow. Suppose $z \in Z$ such that the support $S_{\mu_{1}}$ of $\mu_{t z}$ equals the fiber $\phi_{i}^{-1}(z)$, for $i=1, \ldots, n$. Suppose $X_{0}$ is a minimal flow and $\phi_{0}: X_{0} \rightarrow Z$ is a homomorphism. Given $x$ in $\phi_{0}^{-1}(z) \subseteq X_{0}$ and open sets $V_{i}$ in $\phi_{i}^{-1}(z) \subseteq X_{i}$, the set $N=o c\left(\{x\} \times V_{1} \times \cdots \times V_{n}\right) \supseteq S\left(\phi_{0}\right)(x) v \times$ $Q\left(\phi_{1}\right)\left(V_{1}\right) v \times \cdots \times Q\left(\phi_{n}\right)\left(V_{n}\right) v$ for every $v$ in $J$, and thus $N \supseteq$ $\left[S\left(\phi_{0}\right)(x) v\right] \circ v \times\left[Q\left(\phi_{1}\right)\left(V_{1}\right) v\right] \circ v \times \cdots \times\left[Q\left(\phi_{n}\right)\left(V_{n}\right) v\right] \circ v$.

Proof. Let $v \in J$ with $z v=z$. We will show $N \supseteq S\left(\phi_{0}\right)(x) v \times$ $Q\left(\phi_{1}\right)\left(V_{1}\right) v \times \cdots \times Q\left(\phi_{n}\right)\left(V_{n}\right) v$ by induction. But first some preliminaries. Let $\left(x_{0}, x_{1}, \ldots, x_{n}\right)$ be an element in the right hand side. Then we have $x_{1} v=x_{1}$ and for some $r_{1}$ in $V_{1}, x_{1} \in Q(\phi)\left(r_{1}\right)$. Suppose $v_{1} \in J$ with $r_{1} v_{1}=r_{1}$; define $\alpha: M \rightarrow X_{1}$ by $\alpha(p)=r_{1} p$, then $\phi_{1} \circ \alpha=\psi: M \rightarrow Z$ where $\Psi(p)=z p$. So by 2.3 we see that there exists $p_{1}$ in $S(\psi)(v)=$ $S(\psi)\left(v_{1}\right)$ such that $r_{1} p=\alpha\left(p_{1}\right)=x_{1}$ and $p_{1} v=p_{1}$ since $x_{1} v=x_{1}$. By 2.5 $S\left(\phi_{0}\right)(x) p_{1}=S\left(\phi_{0}\right)(x) v$ and since $x_{0} \in S\left(\phi_{0}\right)(x) v$ there exists $r_{0}$ in $S\left(\phi_{0}\right)(x)$ with $r_{0} p_{1}=x_{0}$, and we may assume $r_{0} v_{1}=r_{0}$. Now (a) $N \supseteq$ $S\left(\phi_{0}\right)(x) \times V_{1} \times \cdots \times V_{n}$ by 1.4 of [6] and so $N \supseteq\left\{\left(r_{0}, r_{1}\right)\right\} \times$ $V_{2} \times \cdots \times V_{n}$. Now consider the flow $o c\left(r_{0} v_{1}, r_{1} v_{1}\right)$. It is minimal and has an induced map $\hat{\phi}: o c\left(r_{0} v_{1}, r_{1} v_{1}\right) \rightarrow Z$. Thus by 1.4 of [6] $N \supseteq$ $S(\hat{\phi})\left(r_{0} v_{1}, r_{1} v_{1}\right) \times V_{2} \times \cdots \times V_{n}$ which equals $S(\hat{\phi})\left(x_{0}, x_{1}\right) \times V_{2}$ $\times \cdots \times V_{n}$ since $\left(r_{0} v_{1}, r_{1} v_{1}\right) p_{1}=\left(x_{0}, x_{1}\right)$ and $p_{1} \in S(\psi)(v)$.

Now we note that when $n=1$ we have for any $x_{0} \in S\left(\phi_{0}\right)(x) v$ and $x_{1} \in Q\left(\phi_{1}\right)\left(V_{1}\right) v$, (b) $o c\left(\{x\} \times V_{1}\right) \supseteq S(\tilde{\phi})\left(x_{0}, x_{1}\right) \ni\left(x_{0}, x_{1}\right)$ and so $o c\left(\{x\} \times V_{1}\right) \supseteq S\left(\phi_{0}\right)(x) v \times Q\left(\phi_{1}\right)\left(V_{1}\right) v$.

Proceeding by induction, assume that the theorem is true for $n=k-$ 1 and prove it for $n=k$. With $n=k$, we have for any $x_{0} \in S\left(\phi_{0}\right)(x) v$ and $x_{1} \in Q\left(\phi_{1}\right)\left(V_{1}\right) v$, (c) $o c\left(\{x\} \times V_{1} \times \cdots \times V_{k}\right) \supseteq o c\left(S(\phi)\left(x_{0}, x_{1}\right) \times\right.$ $\left.\left.V_{2} \times \cdots \times V_{k}\right) \supseteq o c\left(\left\{x_{0}, x_{1}\right)\right\} \times V_{2} \times \cdots \times V_{k}\right) \supseteq S(\tilde{\phi})\left(x_{0}, x_{1}\right) v \times$ $Q\left(\phi_{2}\right)\left(V_{2}\right) v \times \cdots \times Q\left(\phi_{k}\right)\left(V_{k}\right) v \supseteq\left\{x_{0}\right\} \times\left\{x_{1}\right\} \times Q\left(\phi_{2}\right)\left(V_{2}\right) v$ $\times \cdots \times Q\left(\phi_{k}\right)\left(V_{k}\right) v$ by induction. And so $o c\left(\{x\} \times V_{1} \times \cdots \times V_{k}\right) \supseteq$ $S\left(\phi_{0}\right)(x) v \times Q\left(\phi_{1}\right)\left(V_{1}\right) v \times Q\left(\phi_{2}\right)\left(V_{2}\right) v \times \cdots \times Q\left(\phi_{k}\right)\left(V_{k}\right) v$; thus the theorem is proved for every $v \in J$ with $z v=z$ and thus for every $v \in J$.

2.12. Theorem. Suppose for $i=0,1, \ldots, n$ that $\phi_{i}: X^{i} \rightarrow Z$ has a RIM, $\mu_{i}$, and $X^{i}$ is minimal. Suppose $z \in Z$ and $X_{0}^{i}=\phi_{i}^{-1}(z)$. Let $J^{*}$ and $J^{\prime}$ be as in 2.9. Let $V^{i}$ be open in $X_{0}^{i}, A^{i}=V^{i} \cap X_{0}^{i} J^{*}$, and $x \in X_{0}^{i}$. Then

$$
\begin{aligned}
N & =o c\left(\{x\} \times A^{1} \times \cdots \times A^{n}\right) \\
& \supseteq Q\left(\phi_{0}\right)(x) v \times Q\left(\phi_{1}\right)\left(A^{1}\right) v \times \cdots \times Q\left(\phi_{n}\right)\left(A^{n}\right) v
\end{aligned}
$$

for every $v$ in $J$. 
Proof. We indicate where the proof differs from the above. Of course $V_{i}$ is replaced by $A^{i}$ and $J$ by $J^{*}$. Statement (a) would read "Now $N \supseteq S\left(\phi_{0}\right)(x) \cap X_{0}^{0} J^{*} \times A^{1} \times \cdots \times A^{n}$ by Proposition 2.9." Note $S\left(\phi_{0}\right)(x) \cap X_{0}^{0} J^{*}=S\left(\phi_{0}\right)(x) J^{*}$. Statement (b) would read “ $o c\left(\{x\} \times A^{1}\right)$ $\supseteq S(\tilde{\phi})\left(x_{0}, x_{1}\right) J^{*} \ni\left(x_{0}, x_{1}\right)$." Statement (c) would read

$$
\text { “oc }\left(\{x\} \times A^{1} \times \cdots \times A^{n}\right) \supseteq o c\left(S(\tilde{\phi})\left(x_{0}, x_{1}\right) J^{*} \times A^{2} \times \cdots \times A^{n}\right) . "
$$

2.13. Corollary. Suppose $\Gamma$ is an index set and for $i \in \Gamma, \phi_{i}: X_{i} \rightarrow Z$ has $a$ RIM and $X_{i}$ is minimal. Suppose $z \in Z$ and $x_{i}, y_{i} \in X_{i}$ with $x_{i}, y_{i} \in X_{0}^{i} J^{*}=\phi_{i}^{-1}(z) J^{*}, x=\left(x_{i}\right) \in \Pi_{i \in \Gamma} X_{i}, y=\left(y_{i}\right) \in \prod_{i \in \Gamma} X_{i}$ where $J^{*}$ is taken as in 2.9. Then $(x, y) \in Q\left(\Pi \phi_{i}\right)$ iff $\left(x_{i}, y_{i}\right) \in Q\left(\phi_{i}\right)$ for every $i$ in $\Gamma$.

Proof. $(\Rightarrow)$ Clear.

$(\Leftrightarrow)$ Suppose $u \in J^{*}$. Let $\prod_{i \in F} U_{i} \times \prod_{i \notin F} X_{i}$ and $\prod_{i \in F} V_{i} \times \prod_{i \notin F} X_{i}$ be neighborhoods of $x$ and $y$ respectively, where $F$ is a finite subset of $\Gamma$. Let $A_{i}=U_{i} \cap X_{0}^{i} J^{*}$ and $B_{i}=V_{i} \cap X_{0}^{i} J^{*}$. Then $N=o c\left(\Pi A_{i} \times \Pi B_{i}\right) \supseteq$ $\Pi Q\left(A_{i}\right) u \times \Pi Q\left(B_{i}\right) u \supseteq \Pi Q\left(x_{i}\right) u \times \Pi Q\left(y_{i}\right) u \ni \Pi\left\{x_{i} u\right\} \times \Pi\left\{x_{i} u\right\} ;$ and the corollary clearly follows.

REMARK. The above was known under various more specialized conditions, see $[\mathbf{1}, 3]$.

2.14. Corollary. Suppose $\phi: X \rightarrow Z$ has a RIM, $\lambda$, let $z \in Z$, $x_{0} \in X_{0} J^{*}=\phi^{-1}(z) J^{*}, \Gamma$ an index set, and $x_{i} \in S(\phi)\left(x_{0}\right) J^{*}, i \in \Gamma$. Then there exist nets $t_{n}$ in $T$ and $x_{i}^{n}$ in $X_{0} J^{*}$ with $x_{i}^{n} \underset{n}{\rightarrow} x_{i}, x_{i}^{n} t_{n} \underset{n}{\rightarrow} x_{0}$ for $i \in \Gamma$, and $x_{0} t_{n} \rightarrow x_{0}$.

Proof. Let $u \in J^{*}$ with $x_{0} u=x_{0}$. For any neighborhood $V_{i}$ of $x_{i}$ in $X_{0}$ let $A_{i}=V_{i} \cap X_{0} J^{*}$. Then

$$
\begin{aligned}
o c\left(\left\{x_{0}\right\} \times \Pi A_{i}\right) & \supseteq Q\left(\phi\left(x_{0}\right) u\right) \times\left(\Pi Q(\phi)\left(A_{i}\right) u\right) \\
& \supseteq Q(\phi)\left(x_{0}\right) \times \Pi Q(\phi)\left(x_{i}\right) u \ni\left\{x_{0}\right\} \times \Pi\left\{x_{0}\right\} ;
\end{aligned}
$$

and the corollary clearly follows.

2.15. Lemma. Suppose $Z$ is a minimal flow and $z_{0} \in Z$. Define $\psi: M \rightarrow Z$ by $p \rightarrow z_{0} p$ and let $M_{0}=\psi^{-1}\left(z_{0}\right)$. Suppose $\psi$ is RIC and has a RIM, $\lambda$. Then there exist $w \in M_{0} \cap J$ such that for $p$ in $\operatorname{cls}\left(M_{0} w\right)$ and $q$ in $Q(\psi)(p)$ there exist nets $p_{n}$ in $M_{0} w$ and $t_{n}$ in $T$ such that $p_{n} \rightarrow p$, $p_{n} t_{n} \rightarrow p, q t_{n} \rightarrow p$. In particular for $p$ in $\operatorname{cls}\left(M_{0} w\right), Q(\psi)(p)=\{q$ : there exist nets $p_{n}$ in $M_{0} w$ and $t_{n}$ in $T$ with $\left.p_{n} \rightarrow p, p_{n} t_{n} \rightarrow p, q t_{n} \rightarrow p\right\}$ $=\bigcap\left\{\operatorname{cls}\left(\beta T(p) \cap \operatorname{cls}\left(M_{0} w\right)\right): \beta=V \times V, V\right.$ an open set in $\left.M\right\} .($ Recall that $S(\psi)=Q(\psi)$ if $\psi$ in RIC, see [9].) 
Proof. Let $S_{\lambda}$ be the support of $\lambda_{z_{0}}$ and $p \in S_{\lambda} \subseteq M_{0}$. Suppose $u \in J$ with $p u=p$ and $q \in M_{0} u$, then $\mu$ defined by $\mu_{z}(A)=\lambda_{z}\left(q p^{-1} A\right)$ is a RIM and $q \in S_{\mu}$. So if $S=\operatorname{cls}\left(\cup\left\{S_{\mu}: \mu\right.\right.$ is a RIM for $\left.\left.\psi\right\}\right)$, then $S=M_{0} J_{1}$ for some subset $J_{1}$ of $J \cap M_{0}$. Now consider the left flow $\left(M_{0} u, S\right)$ with the action being multiplication on the left and $M_{0} u$ is a group given the discrete topology. Then it contains a minimal set $\left(M_{0} u, \operatorname{cls}\left(M_{0} w\right)\right)$ for some $w$ in $J_{1}$.

Suppose $V$ is an open subset of $M_{0}$ and $V \cap M_{0} w \neq \varnothing$. Then there exists a finite set $F$ of $f$ 's in $M_{0} w$ such that $\cup_{f \in F} F\left(V \cap \overline{M_{0} w}\right) \supseteq \overline{M_{0} w}$. Let $B=B_{V}=\operatorname{cls}\left(V \cap \overline{M_{0} w}\right)=\operatorname{cls}\left(V \cap M_{0} w\right)$. Then $\cup_{f \in F} F(B \circ w)=$ $\left[\cup_{f \in F} f B\right] \circ w \supseteq \overline{M_{0} w} \circ w=M_{0}$ since $\psi$ is RIC. So $\cup_{f \in F}(S \cap f(B \circ w))$ $\supseteq S$. So int $(S \cap f(B \circ w)) \neq \varnothing$ for some $f$ in $F$ where the interior is with respect to $S$, and thus $\operatorname{int}(S \cap(B \circ w)) \neq \varnothing$. Let $p \in \overline{M_{0} w}$ and $p^{*} \in$ $\cap_{V \in \Re_{p}} \operatorname{cls} \operatorname{int}\left(S \cap\left(B_{V} \circ w\right)\right)$.

Suppose $q^{*} \in Q(\psi)\left(p^{*}\right)$ and consider

$$
N_{V}=o c\left(\left\{q^{*} w\right\} \times \operatorname{int}\left[S \cap\left(B_{V} \circ w\right)\right]\right)
$$

then by Lemma 2.7

$$
N_{V} \supseteq\left\{p^{*}\right\} \times \operatorname{cls}\left(\operatorname{int}\left[S \cap\left(B_{V} \circ w\right)\right]\right) \ni\left(p^{*}, p^{*}\right) .
$$

Let $U \in \Re_{p^{*}}$. Then there exist $t=t_{V U}$ in $T$ and $r=r_{V U}$ in $S \cap\left(B_{V} \circ w\right)$ such that $q^{*} w t \in U$ and $r t \in U$. Then there exist $s=s_{V U}$ and $m=m_{V U}$ in $V \cap M_{0} w$ such that $q^{*} s$ is near $q^{*} w$ and $m s$ is near $r$; that is, $q^{*} s \in U t^{-1}$ and $m s \in U t^{-1}$. Thus we have nets $m_{V U}$ in $M_{0} w$ and $s_{V U} t_{V U}$ in $T$ with $m_{V U} \rightarrow p, m_{V U} s_{V U} t_{V U} \rightarrow p^{*}$ and $q^{*} s_{V U} t_{V U} \rightarrow p^{*}$ thus $\left(q^{*}, p\right) \in Q(\psi)$.

So we have assumed $\left(p^{*}, q^{*}\right) \in Q(\psi)$ and shown $\left(q^{*}, p\right) \in Q(\psi)$. Now suppose $(p, q) \in Q(\psi)$; we can repeat the preceeding paragraph with $q$ in place of $q^{*}$ to obtain the lemma.

2.16. Proposition. Suppose $\phi: X \rightarrow Z$ is a homomorphism of minimal flows such that the set $D(\phi)$ of almost periodic points in $R(\phi)$ is dense. Let $x_{0} \in X, \phi\left(x_{0}\right)=z_{0}$, and $X_{0}=\phi^{-1}\left(z_{0}\right)$. Then there exists $w \in J$ with $z_{0} w=z_{0}$ such that for $x, y$ in $\operatorname{cl}\left(X_{0} w\right)$ with $y$ in $Q(\phi)(x)$ and for $p \in$ $\operatorname{cls}(M w)$ with $x_{0} p=x$, there exist $q$ in $M$ and nets $p_{n}$ in $M w$ and $t_{n}$ in $T$ such that $x_{0} q=y$ and $p_{n} \rightarrow p, q t_{n} \rightarrow p, p_{n} t_{n} \rightarrow p$.

Proof. Let $X_{0} \in X_{0}=\phi^{-1}\left(z_{0}\right)$. Define $\beta: M \rightarrow X$ by $\beta(p)=x_{0} p$. Let $\psi=\phi \circ \beta: M \rightarrow Z, M_{0}=\psi^{-1}\left(z_{0}\right)$. Take a proximal extension $Z^{*}$ of $Z, \theta$ : $Z^{*} \rightarrow Z$ such that $\psi^{*}: M^{*} \subseteq M \circ{ }^{Z} Z^{*} \rightarrow Z^{*}$ is RIC and has a RIM. Let $z_{0}^{*} \in \theta^{-1}\left(z_{0}\right), M_{0}^{*}=\psi^{*-1}\left(z_{0}^{*}\right)$, and let $w \in J \cap M_{0}^{*}$ as in Lemma 2.15. If $x \in \operatorname{cls}\left(X_{0} w\right)$ and $y \in Q(\phi)(x)$, then by 2.1 .4 of [6], $((x, z),(y, z)) \in$ $Q\left(\phi^{*}\right)$ for some $z$ in $Z^{*}$, and thus $\left(\left(x w, z_{0}\right),\left(y w, z_{0}\right)\right)=((x w, z w)$, $(y w, z w)) \in Q\left(\phi^{*}\right)$. Since $x, y \in \operatorname{cls}\left(X_{0}, w\right),\left(x, z_{0}\right),\left(y, z_{0}\right) \in X^{*}$ and so 
$\left(\left(x, z_{0}\right),\left(x w, z_{0}\right)\right) \in P,\left(\left(y, z_{0}\right),\left(y w, z_{0}\right)\right) \in P$ and $\left(\left(x, z_{0}\right),\left(y, z_{0}\right)\right) \in$ $Q\left(\phi^{*}\right)$. Let $p \in \operatorname{cls}\left(M_{0} w\right)$ with $\left(x_{0}, z_{0}\right) p=\left(x, z_{0}\right)$. By 14.2 of $\left[\mathbf{2}_{\mathrm{b}}\right]$ we can take $q$ in $Q(\psi)(p)$ with $\left(x_{0}, z_{0}\right) q=\left(y, z_{0}\right)$. The proposition clearly follows from Lemma 2.15 .

A stronger result can be obtained if we assume $Z$ is a singleton. Fix $x_{0}$ in $X$ and define $\psi: M \rightarrow X$ by $p \rightarrow x_{0} p$. Let $u \in J$. Then $M u$ is a group. Give it the discrete topology and consider the (left) flow $(M u, M)$ with the action being multiplication on the left. Then it contains a minimal set $(M u, \overline{M w})$ for some $w$ in $J \subseteq M$. Note $(M w, \overline{M w})$ is also minimal. See 2.10 of $[8]$ for related results.

2.17. Theorem. Suppose $X$ is a minimal flow and has an invariant measure. Let $w \in J$ such that $(M w, \overline{M w})$ is a minimal (left) flow as above. Let $x \in X$. Suppose $x_{0} \overline{M w} \circ w=X$, (that is, $X$ is incompressible). Then for each $x$ in $\overline{X w}=x_{0} \overline{M w}, p$ in $\psi^{-1}(x) \cap \overline{M w}$ and $x^{\prime}$ in $Q(x)$, there exist nets $m_{n}$ in $M w$ and $t_{n}$ in $T$ with $m_{n} \rightarrow p, x_{0} m_{n} \rightarrow x_{0} p=x, x^{\prime} t_{n} \rightarrow x^{*}, x_{0} m_{n} t_{n} \rightarrow$ $x^{*}$ for any $x^{*}$ in $X$. In particular, for $x$ in $\overline{X w}$,

$$
\begin{aligned}
Q(x) & =\left\{x^{\prime}: \text { there exist nets } x_{n} \text { in } X w \text { and } t_{n} \text { in } T\right. \\
& \text { with } \left.x_{n} \rightarrow x, x_{n} t_{n} \rightarrow x, x^{\prime} t_{n} \rightarrow x\right\} \\
& =\cap\{\operatorname{cls}(\alpha T(x) \cap X w): \alpha=V \times V, V \text { an open set in } X\} \\
& =\cap\{\operatorname{cls}(\alpha T(x) \cap \overline{X w}): \alpha=V \times V, V \text { an open set in } X\} .
\end{aligned}
$$

Proof. Suppose $x \in x_{0} \overline{M w}, p \in \psi^{-1}(x) \cap \overline{M w}$ and $V \in \Re_{p}$. Then $V \cap \overline{M w} \neq \varnothing$ and is open in $\overline{M w}$. Then since $(M w, \overline{M w})$ is minimal, there exists a finite set $F$ of $f$ 's in $M w$ such that $\bigcup_{f \in F} f(V \cap \overline{M w}) \supseteq \overline{M w}$. Let $B=B_{v}=\overline{V \cap \overline{M w}}$. Then $\cup_{f \in F} x_{0} f B \circ w=x_{0} \cup_{f \in F} f B \circ w=$ $x_{0}\left[\cup_{f \in F} f B\right] \circ w \supseteq x_{0} \overline{M w} \circ w=X$. So $\operatorname{int}\left(x_{0} f B \circ w\right) \neq \varnothing$ for some $f$ in $F$. Then $\operatorname{int}(B \circ w) \neq \varnothing$. Therefore $\operatorname{int}\left(x_{0} B \circ w\right) \neq \varnothing$. Let $x^{*} \in$ $\cap_{V \in \mathscr{T}_{p}} \operatorname{cls} \operatorname{int}\left(x_{0} B_{V} \circ w\right)$.

Suppose $x^{\#} \in Q\left(x^{*}\right)$ and consider $N_{V}=o c\left(\left\{x^{\#} w\right\} \times \operatorname{int}\left(x_{0} B_{v} \circ w\right)\right)$. Then by 1.4 of [6],

$$
N_{V} \supseteq\left\{x^{*}\right\} \times \operatorname{clsint}\left[x_{0} B_{v} \circ w\right] \ni\left(x^{*}, x^{*}\right) .
$$

Let $U \in \Re_{x^{*}}$. Then there exists $t=t_{V, U}$ in $T$ and $y=y_{V, U}$ in $X_{0} B_{V} \circ w$ such that $x^{\#} w t \in U$ and $y t \in U$. Then there exists $s=s_{V, U}$ in $T$ and $m=m_{V, U}$ in $V \cap M w$ such that $x^{\#}{ }_{s} \in U t^{-1}$ and $x_{0} m s \in U t^{-1}$. Thus we have nets $m_{V, U}$ in $M w$ and $s_{V, U} t_{V, U}$ in $T$ with $m_{V, U} \rightarrow p, x_{0} m_{V, U} \rightarrow x_{0} p$, $x_{0} m_{U, V} s_{V, U} t_{V, u} \rightarrow x^{*}, x^{\#} s_{V, U} t_{V, U} \rightarrow x^{*}$. Thus $\left(x^{\#}, x\right)=\left(x^{\#}, x_{0} p\right) \in Q$. 
So we have assumed $\left(x^{*}, x^{\#}\right) \in Q$ and shown $\left(x^{\#}, x\right) \in Q$. Now suppose $\left(x, x^{\prime}\right) \in Q$; then $\left(x^{*}, x^{\prime}\right) \in Q$ and we can repeat the preceding paragraph with $x^{\prime}$ in place of $x^{\#}$ to obtain the theorem (note the $x^{*}$ can be replaced as the limit by any point in $X$ since $X$ is a minimal flow).

\section{REFERENCES}

1. Jesse P. Clay, Proximity relations in transformation groups, Trans. Amer. Math. Soc., 108 (1963), 88-96.

2. Robert Ellis, Lectures on Topological Dynamics, Benjamin, New York, 1969.

3. R. Ellis and H. B. Keynes, A characterization of the equicontinuous structure relation, Trans. Amer. Math. Soc., 161 (1971), 171-183.

4. Samuel Glasner, Relatively invariant measure, Pacific J. Math., 58 (1975), 393-417.

5. __ Proximal flows, Lecture Notes in Math., Vol. 517, Springer-Verlag, Berlin and New York, 1976.

6. Doug McMahon, Relativized weak disjointness and relatively invariant measures, Trans. Amer. Math. Soc., 236 (1978), 225-237.

7. $559-566$.

, Relativized weak disjointness of uncountable order, Canad. J. Math., 32 (1980),

8. D. McMahon and T. S. Wu, On proximal and distal extensions of minimal sets, Bull. Inst. Math. Acad. Sinica, 2 (1974), no. 1, 93-107.

9. W. A. Veech, Topological dynamics, Bull. Amer. Math. Soc., 83 (1977), 775-830.

10. Point-distal flows, Amer. J. Math., 92 (1970), 205-242.

Received April 9, 1981.

ARIZONa State UnIVERSITY

TEMPE, AZ 85287

AND

CASE Western Reserve University

Cleveland, OH 44106 


\title{
PACIFIC JOURNAL OF MATHEMATICS EDITORS
}

\author{
Donald BABBITT (Managing Editor) \\ University of California \\ Los Angeles, CA 90024 \\ Hugo Rossi \\ University of Utah \\ Salt Lake City, UT 84112 \\ C. C. Moore and Arthur Ogus \\ University of California \\ Berkeley, CA 94720
}

\author{
J. Dugundi \\ Department of Mathematics \\ University of Southern California \\ Los Angeles, CA 90089-1113
}

R. FinN and H. SAMELSON

Stanford University

Stanford, CA 94305

\section{ASSOCIATE EDITORS}
R. ARENS
E. F. BECKENBACH
B. H. NeumanN
F. WOLF
K. YoSHIDA (1906-1982)

\section{SUPPORTING INSTITUTIONS}

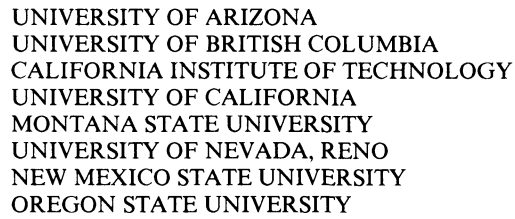

UNIVERSITY OF ARIZONA

UNIVERSITY OF BRITISH COLUMBIA

CALIFORNIA INSTITUTE OF TECHNOLOGY

UNIVERSITY OF CALIFORNIA

MONTANA STATE UNIVERSITY

UNIVERSITY OF NEVADA, RENO

NEW MEXICO STATE UNIVERSITY

OREGON STATE UNIVERSITY

\author{
UNIVERSITY OF OREGON \\ UNIVERSITY OF SOUTHERN CALIFORNIA \\ STANFORD UNIVERSITY \\ UNIVERSITY OF HAWAII \\ UNIVERSITY OF TOKYO \\ UNIVERSITY OF UTAH \\ WASHINGTON STATE UNIVERSITY \\ UNIVERSITY OF WASHINGTON
}

The Supporting Institutions listed above contribute to the cost of publication of this Journal, but they are not owners or publishers and have no responsibility for its content or policies.

Mathematical papers intended for publication in the Pacific Journal of Mathematics should be in typed form or offset-reproduced (not dittoed), double spaced with large margins. Please do not use built up fractions in the text of the manuscript. However, you may use them in the displayed equations. Underline Greek letters in red, German in green, and script in blue. The first paragraph must be capable of being used separately as a synopsis of the entire paper. In particular it should contain no bibliographic references. Please propose a heading for the odd numbered pages of less than 35 characters. Manuscripts, in triplicate, may be sent to any one of the editors. Please classify according to the scheme of Math. Reviews, Index to Vol. 39. Supply name and address of author to whom proofs should be sent. All other communications should be addressed to the managing editor, or Elaine Barth, University of California, Los Angeles, California 90024.

There are page-charges associated with articles appearing in the Pacific Journal of Mathematics. These charges are expected to be paid by the author's University, Government Agency or Company. If the author or authors do not have access to such Institutional support these charges are waived. Single authors will receive 50 free reprints; joint authors will receive a total of 100 free reprints. Additional copies may be obtained at cost in multiples of 50 .

The Pacific Journal of Mathematics is issued monthly as of January 1966. Regular subscription rate: $\$ 132.00$ a year (6 Vol., 12 issues). Special rate: $\$ 66.00$ a year to individual members of supporting institutions.

Subscriptions, orders for numbers issued in the last three calendar years, and changes of address should be sent to Pacific Journal of Mathematics, P.O. Box 969, Carmel Valley, CA 93924, U.S.A. Old back numbers obtainable from Kraus Periodicals Co., Route 100, Millwood, NY 10546.

The Pacific Journal of Mathematics ISSN 0030-8730 is published monthly by the Pacific Journal of Mathematics at P.O. Box 969, Carmel Valley, CA 93924. Application to mail at Second-class postage rates is pending at Carmel Valley, California, and additional mailing offices. Postmaster: Send address changes to Pacific Journal of Mathematics, P. O. Box 969, Carmel Valley, CA 93924.

PUBLISHED BY PACIFIC JOURNAL OF MATHEMATICS, A NON-PROFIT CORPORATION

Copyright $\odot 1983$ by Pacific Journal of Mathematics 


\section{Pacific Journal of Mathematics}

Vol. 104, No. $2 \quad$ June, 1983

Leo James Alex, Simple groups and a Diophantine equation ........... 257

Herbert James Alexander and John Wermer, On the approximation of

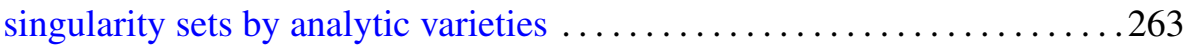

Waleed A. Al-Salam and Mourad Ismail, Orthogonal polynomials associated with the Rogers-Ramanujan continued fraction .......... 269

J. L. Brenner and Roger Conant Lyndon, Permutations and cubic

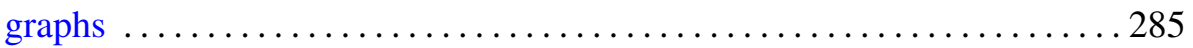

Ian George Craw and Susan Ross, Separable algebras over a commutative

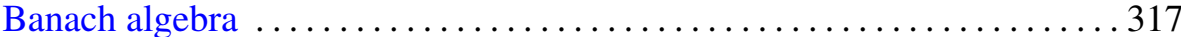

Jesus M. Dominguez, Non-Archimedean Gel'fand theory ............. 337

David Downing and Barry Turett, Some properties of the characteristic of

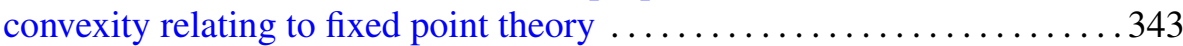

James Arthur Gerhard and Mario Petrich, Word problems for free objects in certain varieties of completely regular semigroups $\ldots \ldots \ldots 351$

Moses Glasner and Mitsuru Nakai, Surjective extension of the reduction

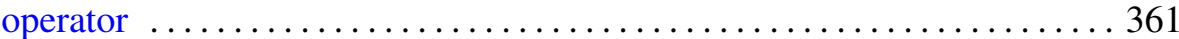

Takesi Isiwata, Ultrafilters and mappings $\ldots \ldots \ldots \ldots \ldots \ldots \ldots \ldots \ldots \ldots \ldots$

Lowell Duane Loveland, Double tangent ball embeddings of curves in $E^{3}$

Douglas C. McMahon and Ta-Sun Wu, Homomorphisms of minimal flows

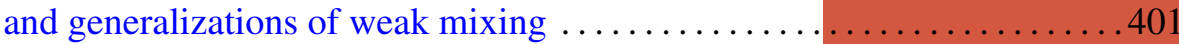

P. H. Maserick, Applications of differentiation of $\mathscr{L}_{p}$-functions to semilattices

Wayne Bruce Powell and Constantine Tsinakis, Free products in the class of abelian $l$-groups

Bruce Reznick, Some inequalities for products of power sums

C. Ray Rosentrater, Compact operators and derivations induced by

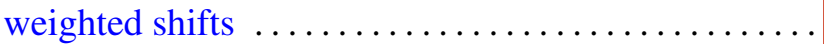

Edward Silverman, Basic calculus of variations

Charles Andrew Swanson, Criteria for oscillatory sublinear Schrödinger equations

David J. Winter, The Jacobson descent theorem 